\title{
Collagen cross-linking: Strengthening the unstable cornea
}

\author{
Oren Tomkins \\ Hanna J Garzozi \\ Department of Ophthalmology, \\ Bnai Zion Medical Center, Haifa, Israel
}

\begin{abstract}
Corneal ectasia, a weakening of corneal integrity, occurs both due to acquired and congenital conditions such as keratoconus. It is a progressing condition that affects both visual acuity, and corneal stability. Various methods exist for correcting this impairment, however none address the inherit pathology, an increase laxity of the corneal stroma. Collagen crosslinking, a new, minimally invasive method, aims to strengthen the stroma by inducing cross links between neighboring collagen fibers. This method results in an increase in corneal tensile strength, with no medium term adverse effects on its normal architecture. Clinically, treated patients display improvement in both visual acuity and keratometric readings. This method may provide clinicians with easily accessible tools to stop the progression, and even correct visual deterioration due to corneal ectasia. Here we review the current information regarding this new method, as well as discuss its potential benefits and downfalls.
\end{abstract}

Keywords: corneal cross-linking, corneal ectasia, keratoconus, stroma, cornea

\section{Introduction}

The cornea, the anterior structure of the eye, is a convex transparent barrier. It serves to maintain the intact structure of the eye and focus a clear and minimally deviated penetration of light onto the retina. It consists of five anatomically separate layers: epithelium; Bowmen's layer; stroma; Descement's membrane; and endothelium. The stroma accounts for $90 \%$ of the corneal thickness. It is composed mainly of type 1 collagen fibrils with types III, V, and VI also found. These are woven into equidistant lamellae, interspersed with keratocytes. These are charged with stromal maintenance and wound healing (Watsky et al 2005).

Corneal ectasia represents a group of disorders characterized by inherent corneal weakness and instability leading to protrusion, astigmatism, loss of visual acuity, and potentially even perforation (Krachmer et al 1984). This entity comprises of both primary and secondary or iatrogenic conditions, all leading to similar manifestations of loss of corneal stability. Of the congenital disorders, keratoconus represents the primary cause, affecting up to one in 2000 people (Rabinowitz 1998). This congenital, noninflammatory, usually bilateral disease manifests as a progressing stromal instability, leading to a reduction in corneal rigidity (Andreassen et al 1980) and may affect visual acuity to such an extent as to require penetrating keratoplasy in up to $20 \%$ of patients (Pramanik et al 2006). Treatment options include conservative approaches, aimed at maintaining visual acuity, such as rigid contact lenses placed to straighten corneal aberrations (Garcia-Lledo et al 2006). Alternatively, more invasive methods are applied using intrastromal corneal implants (Ertan and Colin 2007), performing anterior lamellar keratoplasty, and penetrating keratoplasty in extreme cases (Pramanik et al 2006; Tan and Por 2007). However, these therapies only address the refractive consequences of the disease and not the underlying pathology, namely a stromal instability stemming from collagen abnormalities (Rabinowitz 1998), and 
with the exception of corneal transplantation do not halt the progression of the disease.

Collagen cross-linking is a new approach that directly targets these stromal imbalances. By using ultraviolet (UV) irradiation and a photomediator, new covalent cross links are induced between collagen fibrils and corneal rigidity improved (Wollensak 2006). Cross-linking is a well established method used in polymer industries for the stabilization of tissues. Chemical cross-linking with glutaraldehyde is used in the preparation of prosthetic heart valves (Jayakrishnan and Jameela 1996), papraformaldehyde is used for tissue hardening in pathology (Melan 1994) and UV cross-linking is used to harden dentistry fillings (de Gee et al 1998). Furthermore, studies have shown that keratoconus is an uncommon phenomenon among diabetic patients, as well as progression of the disease at an older age. This possibly reflects collagen cross-linking induced by advanced glycation endproducts (Bailey et al 1995; Seiler et al 2000; Krueger and Ramos-Esteban 2007), or following age-related cross-linking (Reiser 1991; Malik et al 1992; Elsheikh et al 2007).

\section{Method}

Inducing cross links between neighboring collagen fibers is achieved by combining the photosensitizer riboflavin with an initiating UVA beam. Exposing riboflavin to UVA light promotes its photomediator properties and extends the effects of the irradiation to the surrounding tissue. Following exposure, riboflavin is excited into a triplet state thereby generating reactive oxygen species; singlet oxygen and superoxide anions. These then act to induce the formation of new covalent bonds between the amino acids of neighboring collagen fibers (Seiler et al 2000). The wavelength of $370 \mathrm{~nm}$ is used to achieve maximal absorption by the riboflavin, while remaining below harmful DNA and retinal radiation levels (Spoerl et al 2007).

The treatment is conducted following local anesthesia of the eye. An abrasion of a central corneal circle is created by mechanical removal of the epithelium for better riboflavin diffusion into the stroma. This is made at a size of between 5-9 $\mathrm{mm}$ according to the desired treatment area (Caporossi et al 2006; Hafezi et al 2007; Kanellopoulos and Binder 2007). A $0.1 \%$ riboflavin solution in $20 \%$ dextran is applied manually every 2-5 minutes, starting 5 minutes before UVA exposure to allow stromal saturatuion. The irradiation is performed from a distance of $1 \mathrm{~cm}$ for 30 minutes. A UVA diode at a wave length of $365-370 \mathrm{~nm}$ is used to deliver an irradiance of $3 \mathrm{~mW} / \mathrm{cm}^{2}$ (a total dose of $5.4 \mathrm{~J} / \mathrm{cm}^{2}$ of the cornea) (Wollensak et al 2003a; Seiler and Hafezi 2006). Repeated applications of riboflavin and topical anesthesia to the cornea are performed every 5 minutes. Corneal temperature during the procedure is constant and does not cause thermal damage (Mencucci et al 2007). It should be noted that alternative protocols have been reported using irradiances distances of up to $10 \mathrm{~cm}$ (Hafezi et al 2007; Mazzotta et al 2007b), riboflavin application up to 30 minutes before irradiation (Hafezi et al 2007; Spoerl et al 2007) and repeated applications of physiological salt solutions to maintain corneal moisture (Hafezi et al 2007; Kymionis et al 2007b). Following the treatment a contact lens is fitted until re-epitalization and local antibiotics and steroids are applied for a duration of up to several weeks (Wollensak et al 2003a; Hafezi et al 2007; Spoerl et al 2007).

\section{Discussion}

The efficacy and safety of this new, minimally invasive method for correcting corneal ectasia has been the focus of recent studies. Most work has focused around keratoconus patients, while more recent reports have been published on iatrogenic corneal ectasia as following laser-assisted in situ keratomileusis (LASIK) (Hafezi et al 2007; Kohlhaas et al 2005).

To examine the change in corneal rigidity stress-strain tests were conducted on porcine corneas. These tests showed that collagen cross-linking by glutaraldehyde, Karnovsky's solution, or riboflavin-UV irradiation increased the rigidity of the treated corneas compared with controls (Spoerl et al 1998; Spoerl and Seiler 1999). The treated porcine corneas showed an average increase in rigidity of $71.9 \%$, while stiffness of treated human corneas increased by a mean of $328.9 \%$ (Wollensak et al 2003b).

Although the treatment increased the mechanical strain of the cornea, its effect was not uniform through its entire depth. Stress-strain tests conducted on anterior and posterior corneal flaps showed a significant increase in corneal stiffness in the anterior $200 \mu \mathrm{m}$ compared with the posterior $200 \mu \mathrm{m}$. The stiffness of the posterior treated sections showed no difference from that of untreated controls (Kohlhaas et al 2006). Furthermore, histological sections of treated corneas revealed that the treatment effected the cornea up to a depth of $300 \mu \mathrm{m}$, creating a clinically visible demarcation line separating the anterior cross-linked stroma and the posterior unaffected cornea (Seiler and Hafezi 2006). This suggests that the UVA irradiation is lost across the cornea and does not penetrate its full depth, possibly due to a shielding effect of the photosensitizer riboflavin that absorbs up to $95 \%$ of the UVA radiation at the endothelium level (Spoerl et al 2007). Thus, a constant 
depth of effect is achieved with no disturbance to underlying structures including the endothelium. This may also explain the difference in increased rigidity observed between human and porcine corneas. Whereas in human corneas over $50 \%$ of the corneal thickness was effected (central corneal thickness was measured as $550 \pm 40 \mu \mathrm{m}$ ), under $40 \%$ of the porcine corneas was treated (central corneal thickness of $850 \pm 70 \mu \mathrm{m}$ ) (Wollensak et al 2003b). Such cross-linked corneas also displayed increased resistance to collagen enzymatic ingestion (Spoerl et al 2004b) and thermal shrinkage (Spoerl et al 2004a), further indicating an increased stiffness.

To explore the cellular effects of this treatment, histological sections were obtained from both treated animal and human corneas. Measurements of anterior section collagen diameter of treated rabbit corneas showed an increase of $12.2 \%$ compared to controls. This was also significantly greater than an increase of $4.6 \%$, noted in posterior sections (Wollensak et al 2004). Such increased diameter may reflect a molecular spacing of the collagen, brought on by the oxidative induced links between fibers (Tanaka et al 1988). Histological evaluation further revealed a delayed process of keratocyte apoptosis, peaking at 24 hours following treatment. The extent and depth of cell death were dose-dependent, reaching $300 \mu \mathrm{m}$ at the clinically used surface irradiance of $3 \mathrm{~mW} / \mathrm{cm}^{2}$ (Wollensak et al 2004), and correlating to the affected zone according to the tensile studies. Such delayed type apoptosis was observed at all treated levels, even at the endothelial cell layer when treatment was directed at rabbit corneas under $400 \mu \mathrm{m}$ thickness (Wollensak et al 2003b). Serial histological follow-up of treated rabbit corneas revealed that following such extensive apoptosis, a regeneration process is initiated, leading by $4-6$ weeks to a repopulation of both stromal keratocyes and spacing out of endothelial cells (Wollensak et al 2007). Confocal microscopy studies conducted on corneas of human keratoconus patients showed a depletion of keratocytes in the treated zone up to a depth of $350 \mu \mathrm{m}$, not affecting the endothelium (Mazzotta et al 2007b). The numbers of keratocytes decreased during the first 2-3 months following treatment, when a repopulation by activated keratocytes took place, peaking at 6 months. In these patients, keratocyte depletion was accompanied by an increased corneal edema, which resolved with keratocyte repopulation.

Thus, these studies show that while collagen cross-linking induces cellular apoptosis in all treated layers, later regeneration processes result in repopulation and normal tissue histology. However, it should be noted that as endothelial cells do not regenerate, any damage to the endothelium is irreversible. In cases when the endothelium is affected, as is the concern in patients with corneas under $400 \mu \mathrm{m}$ (Wollensak et al 2003b), the long term consequences of such irreversible damage remain unknown. As the treatment affects a known and constant range, damage to such deeper structures may be averted by maintaining a minimal corneal thickness before treatment.

To date, few studies have examined the effectiveness of collagen cross-linking in treating keratoconus and improving visual acuity. In a prospective study, Wollensak and colleagues (2003a, 2003c) performed collagen cross-linking on 22 moderate to severe keratoconus patients. Follow-up was for up to 4 years (3-47 months) and included visual acuity, corneal topography and endothelial cell counts. In 20 patients refractive follow-up was performed. In 19 patients, $\mathrm{K}$ readings did not progress and in $65 \%$ of cases an average regression of 2.0 diopters was noted. Best corrected visual acuity (BCVA) improved in $65 \%$ of cases by an average of 1.26 lines and the spherical equivalent refractive error by an average of 1.14 diopters. No significant change in endothelial cell density or other adverse reactions were noted. Although follow-up time among the different patients varied, this work was the first to show a significant improvement in the outcome of moderate and severe keratoconus patients following collagen cross-linking treatment. In a second study 10 patients were followed for 6 months after the procedure. In these cases BCVA improved by an average of 1.66 lines with a mean $\mathrm{K}$ readings reduction of 2.1 diopters. As in the former study, no changes were noted in endothelial cell counts or intraocular pressure (Caporossi et al 2006). These first clinical studies provide evidence regarding the medium term benefit of this procedure and also indicate that no other corneal effects take place. Recently a case report has been published of a keratoconus patient, with a BCVA of 20/40, who underwent CCL in one eye (Kanellopoulos and Binder 2007). Twelve months later topography-guided photorefractive keratectomy (PRK) was performed on the same eye. He was followed for another 18 months and his final BCVA was 20/15, with no change to endothelial cell counts. Meanwhile, BCVA of his untreated eye regressed from $20 / 15$ to $20 / 25$.

Recent reports suggest this method may have potential uses also in non keratoconus corneal ectasia (Kohlhaas et al 2005). Hafezi and colleagues (2007) reported a series of 10 patients treated with collagen cross-linking following LASIK-induced keratectasia. Patients were followed for up to 25 months post-treatment. In all cases the progression of corneal ectasia was halted with an improvement in 5 patients 
of over 2 diopters in $\mathrm{K}$ readings. Furthermore, in 4 cases BCVA improved by more than 2 lines.

Though collagen cross-linking is a minimally invasive method, recent reports have indicated possible adverse effects. Mazzotta and colleagues (2007a) have presented two cases of postoperative corneal haze among a cohort of 40 eyes of 39 keratoconus patients. Preoperative BCVA and keratometric readings as well as in vivo confocal examinations were performed on all patients. They report confocal findings which possibly reflect keratoconus. In two cases unchanging stromal haze appeared 2-3 months following the procedure and was resistant to topical steroid treatment. Repeated examination of the preoperative confocal studies of these patients revealed a reticular pattern of stromal microstriae that may imply advanced keratoconus. BCVA in these patients improved despite the haze. Although these confocal findings are not unequivocally due to keratoconus, the authors suggest them to be a relative contra-indication to performing the procedure. Additional case reports describe diffuse lamellar keratitis (Kymionis et al 2007a) and a reactivation of herpetic keratitis (Kymionis et al 2007b) following collagen crosslinking. In both cases prompt diagnosis and treatment resulted in a favorable resolution.

When judging the use of a treatment with the potential of causing damage to ocular structures one must consider its safety and possible harmful consequences. UV radiation is known to be harmful to the endothelium, lens, and retina (Pitts et al 1977; Glickman 2002). Using a wavelength of $360-370 \mathrm{~nm}$ with an accumulated irradiance of $5.4 \mathrm{~J} / \mathrm{cm}^{2}$ ensures that the exposure of all these structures is below harmful levels (Spoerl et al 2007). However, the occurrence of a nonhomogenous irradiation field may create localized hot spots of increased radiance with potential harmful consequences. Therefore, for clinical use, a uniformly emitting irradiance source is needed and must be continuously evaluated. Further protection is achieved by the presence of riboflavin in the cornea, which was shown to reach up to $400 \mu \mathrm{m}$ after 30 minutes of application. This then provides an UV absorption coefficient that shields the more posterior structures including the endothelium (Spoerl et al 2007). When treatment was conducted on corneas of less than $400 \mu \mathrm{m}$, apoptotic damage was noted in the endothelium (Wollensak et al 2003b). This issue may be of greater concern when treating patients following LASIK when the post-treatment effective corneal thickness may very well be under $400 \mu \mathrm{m}$ (Hafezi et al 2007). While the shielding effect and distance from the treated area ensure the lens and retina are exposed to radiation well below harmful levels, the endothelium is still exposed to radiance which is lower than harmful levels only by a factor of $2\left(0.32 \mathrm{~J} / \mathrm{cm}^{2}\right)$ (Spoerl et al 2007). Thus, any variance in the irradiation field or corneal thickness may lead to direct damage to this layer. To ensure that the conducted procedure is safe to all ocular structures it is paramount to maintain a homogenous irradiation field at a wavelength and irradiance below harmful levels, good stromal penetration of riboflavin and adequate corneal thickness. For a more detailed review of the safety considerations of collagen cross-linking see Spoerl and colleagues (2007).

Our personal experience with collagen cross-linking includes 70 patients treated during the past 2 years. Most patients suffered from keratoconus $(n=68), 12$ treated following the insertion of intra-stromal corneal rings. The remaining patients $(n=2)$ were treated for corneal ectasia following LASIK surgery. Our overall impression in these cases is of an improvement in $\mathrm{K}$ readings and BCVA, with no adverse events noted to date.

\section{Conclusions}

Keratoconus, and other corneal ectasias, present us with a treatment challenge in the hope of halting their progression and possibly correcting any refractive and stability deterioration. Studies in both animal models and human patients indicate that collagen cross-linking results in an increase in corneal stiffness and improvement of visual acuity. Furthermore, although the method affects the cellular composition of irradiated structures no lasting effect is generally noted in corneal architecture. Collagen cross-linking provides us for the first time with a minimally invasive approach to addressing the underlying pathology in corneal ectasia, halting its progression, and possibly even improving visual acuity. While it may halt progression of corneal ectasia, collagen cross-linking does not in itself affect corneal curvature, and thus may require the use of other means, such as intrastromal corneal rings (Ertan and Colin 2007) or rigid contact lenses (Garcia-Lledo et al 2006) to correct refractive errors (Tan and Por 2007). Though this method appears to offer an improvement to patients suffering from corneal ectasia, it must be noted that its safety and long-term effects have not been extensively studied. The turnover rate of collagen fibers in the cornea is several years (Ihanamaki et al 2001) and it remains unclear whether the changes noted in corneal stability will last or whether its effects are limited. Furthermore, the effect of corneal thickness on endothelial and ocular damage remains a safety concern. Future studies are needed to explore these long-term effects as well as other aspects of ocular safety. 


\section{Disclosure}

The authors report no conflicts of interest.

\section{References}

Andreassen TT, Simonsen AH, Oxlund H. 1980. Biomechanical properties of keratoconus and normal corneas. Exp Eye Res, 31:435-41.

Bailey AJ, Sims TJ, Avery NC, et al. 1995. Non-enzymic glycation of fibrous collagen: reaction products of glucose and ribose. Biochem $J$, 305(Pt 2):385-90.

Caporossi A, Baiocchi S, Mazzotta C, et al. 2006. Parasurgical therapy for keratoconus by riboflavin-ultraviolet type A rays induced cross-linking of corneal collagen: preliminary refractive results in an Italian study. $J$ Cataract Refract Surg, 32:837-45.

de Gee AJ, Leloup G, Werner A, et al. 1998. Structural integrity of resinmodified glass ionomers as affected by the delay or omission of light activation. J Dent Res, 77:1658-63.

Elsheikh A, Wang D, Brown M, et al. 2007. Assessment of corneal biomechanical properties and their variation with age. Curr Eye Res, 32:11-19.

Ertan A, Colin J. 2007. Intracorneal rings for keratoconus and keratectasia. $J$ Cataract Refract Surg, 33:1303-14.

Garcia-Lledo M, Feinbaum C, Alio JL. 2006. Contact lens fitting in keratoconus. Compr Ophthalmol Update, 7:47-52.

Glickman RD. 2002. Phototoxicity to the retina: mechanisms of damage. Int J Toxicol, 21:473-90.

Hafezi F, Kanellopoulos J, Wiltfang R, et al. 2007. Corneal collagen crosslinking with riboflavin and ultraviolet A to treat induced keratectasia after laser in situ keratomileusis. J Cataract Refract Surg, 33:2035-40.

Ihanamaki T, Salminen H, Saamanen AM, et al. 2001. Age-dependent changes in the expression of matrix components in the mouse eye. Exp Eye Res, 72:423-31.

Jayakrishnan A, Jameela SR. 1996. Glutaraldehyde as a fixative in bioprostheses and drug delivery matrices. Biomaterials, 17:471-84.

Kanellopoulos AJ, Binder PS. 2007. Collagen cross-linking (CCL) with sequential topography-guided PRK: a temporizing alternative for keratoconus to penetrating keratoplasty. Cornea, 26:891-5.

Kohlhaas M, Spoerl E, Schilde T, et al. 2006. Biomechanical evidence of the distribution of cross-links in corneas treated with riboflavin and ultraviolet A light. J Cataract Refract Surg, 32:279-83.

Kohlhaas M, Spoerl E, Speck A, et al. 2005. A new treatment of keratectasia after LASIK by using collagen with riboflavin/UVA light cross-linking. Klin Monatsbl Augenheilkd, 222:430-6.

Krachmer JH, Feder RS, Belin MW. 1984. Keratoconus and related noninflammatory corneal thinning disorders. Surv Ophthalmol, 28:293-322.

Krueger RR, Ramos-Esteban JC. 2007. How might corneal elasticity help us understand diabetes and intraocular pressure? J Refract Surg, 23:85-8.

Kymionis GD, Bouzoukis DI, Diakonis VF, et al. 2007a. Diffuse lamellar keratitis after corneal crosslinking in a patient with post-laser in situ keratomileusis corneal ectasia. J Cataract Refract Surg, 33:2135-7.

Kymionis GD, Portaliou DM, Bouzoukis DI, et al. 2007b. Herpetic keratitis with iritis after corneal crosslinking with riboflavin and ultraviolet A for keratoconus. J Cataract Refract Surg, 33:1982-4.

Malik NS, Moss SJ, Ahmed N, et al. 1992. Ageing of the human corneal stroma: structural and biochemical changes. Biochim Biophys Acta, 1138:222-8.
Mazzotta C, Balestrazzi A, Baiocchi S, et al. 2007a. Stromal haze after combined riboflavin-UVA corneal collagen cross-linking in keratoconus: in vivo confocal microscopic evaluation. Clin Exper Ophthalmol, 35:580-2.

Mazzotta C, Balestrazzi A, Traversi C, et al. 2007b. Treatment of progressive keratoconus by riboflavin-UVA-induced cross-linking of corneal collagen: ultrastructural analysis by Heidelberg Retinal Tomograph II in vivo confocal microscopy in humans. Cornea, 26:390-7.

Melan MA. 1994. Overview of cell fixation and permeabilization. Methods Mol Biol, 34:55-66.

Mencucci R, Mazzotta C, Rossi F, et al. 2007. Riboflavin and ultraviolet A collagen crosslinking: in vivo thermographic analysis of the corneal surface. J Cataract Refract Surg, 33:1005-8.

Pitts DG, Cullen AP, Hacker PD. 1977. Ocular effects of ultraviolet radiation from 295 to $365 \mathrm{~nm}$. Invest Ophthalmol Vis Sci, 16:932-9.

Pramanik S, Musch DC, Sutphin JE, et al. 2006. Extended long-term outcomes of penetrating keratoplasty for keratoconus. Ophthalmology, 113:1633-8

Rabinowitz YS. 1998. Keratoconus. Surv Ophthalmol, 42:297-319.

Reiser KM. 1991. Nonenzymatic glycation of collagen in aging and diabetes. Proc Soc Exp Biol Med, 196:17-29.

Seiler T, Hafezi F. 2006. Corneal cross-linking-induced stromal demarcation line. Cornea, 25:1057-9.

Seiler T, Huhle S, Spoerl E, et al. 2000. Manifest diabetes and keratoconus: a retrospective case-control study. Graefes Arch Clin Exp Ophthalmol, 238:822-5.

Spoerl E, Huhle M, Seiler T. 1998. Induction of cross-links in corneal tissue. Exp Eye Res, 66:97-103.

Spoerl E, Mrochen M, Sliney D, et al. 2007. Safety of UVA-riboflavin cross-linking of the cornea. Cornea, 26:385-9.

Spoerl E, Seiler T. 1999. Techniques for stiffening the cornea. $J$ Refract Surg, 15:711-13.

Spoerl E, Wollensak G, Dittert DD, et al. 2004a. Thermomechanical behavior of collagen-cross-linked porcine cornea. Ophthalmologica, 218:136-40.

Spoerl E, Wollensak G, Seiler T. 2004b. Increased resistance of crosslinked cornea against enzymatic digestion. Curr Eye Res, 29:35-40.

Tan DT, Por YM. 2007. Current treatment options for corneal ectasia. Curr Opin Ophthalmol, 18:284-9.

Tanaka S, Avigad G, Brodsky B, et al. 1988. Glycation induces expansion of the molecular packing of collagen. $J$ Mol Biol, 203:495-505.

Watsky MT, Olsen TW, Edelhauser HF. 2005. Cornea and sclera. In: Tasman W (ed). Duane's Clinical Ophthalmology. Philadelphia, PA: Lippincott Williams and Wilkins.

Wollensak G. 2006. Crosslinking treatment of progressive keratoconus: new hope. Curr Opin Ophthalmol, 17:356-60.

Wollensak G, Iomdina E, Dittert DD, et al. 2007. Wound healing in the rabbit cornea after corneal collagen cross-linking with riboflavin and UVA. Cornea, 26:600-5.

Wollensak G, Spoerl E, Seiler T. 2003a. Riboflavin/ultraviolet-a-induced collagen crosslinking for the treatment of keratoconus. Am J Ophthalmol, 135:620-7.

Wollensak G, Spoerl E, Seiler T. 2003b. Stress-strain measurements of human and porcine corneas after riboflavin-ultraviolet-A-induced cross-linking. J Cataract Refract Surg, 29:1780-5.

Wollensak G, Sporl E, Seiler T. 2003c. [Treatment of keratoconus by collagen cross linking]. Ophthalmologe, 100:44-9.

Wollensak G, Wilsch M, Spoerl E, et al. 2004. Collagen fiber diameter in the rabbit cornea after collagen crosslinking by riboflavin/UVA. Cornea, 23:503-7. 
